\title{
Abrasive Wear Behavior of Different Thermal Spray Coatings and Hard Chromium Electroplating on A286 Super Alloy ${ }^{*}$
}

\author{
Macid Nurbaș, Elif Nazik Atabay Durul \\ ${ }^{1}$ ESOGU, Chemical Eng. Dept. Eskisehir, Turkey \\ ${ }^{2}$ Air Supply and Maintenance Center Command Eskisehir, Turkey \\ Email: mnurbas@gmail.com, eatabay@hvkk.tsk.tr
}

Received 2012

\begin{abstract}
In cases of decorative and functional applications, chromium results in protection against wear and corrosion combined with chemical resistance and good lubricity. However, pressure to identify alternatives or to improve conventional chromium electroplating mechanical characteristics has increased in recent years, related to the reduction in the fatigue strength of the base material and to environmental requirements (1). In the present study plasma sprayed coatings (aluminum oxide, Co-28Mo-8Cr-2Si, tungsten carbide, chrome carbide) and electrolytic hard chrome coatings abrasive wear properties have been compared. The wear tests were conducted with a Taber abraser, at room temperature.
\end{abstract}

Keywords: Thermal Spray; Abrasive Wear; Electrodeposited Hard Chrome; Hardness; SEM

\section{Introduction}

Chromium has been widely used in surface finishing of metals because of the favorable properties it imparts to substrates and because the processes used are relatively mature, well understood, widely specified, and cost effective (2). This coating is produced from a wet chemical bath containing hexavalent chromium ions $\left(\mathrm{Cr}^{+6}\right)$. In all environmental regulations, $\mathrm{Cr}^{+6}$ is classified as a confirmed human carcinogen. Hard chromium plating produces large volumes of chromium containing toxic waste, air pollution and water contamination (3). Potential process substitutions for hard chromium plating are electroless nickel in certain applications, several nickel-tungsten composite plating, and spray applications such as plasma spray coatings (2). Plasma spraying is a process widely used in industry for depositing protective and functional coatings for a large variety of applications. Industrial sectors such as aerospace, automotive, energy, mining, biomedical, etc. take advantage of the unique properties of the sprayed coatings (4). The applications of thermal spray coatings are extremely varied, but the largest categories of use are to enhance the wear and/or corrosion resistance of a surface (2). The deposition methods for the wear protective coatings are atmospheric plasma spraying (APS) and high velocity oxygen fuel (HVOF) flame spray processes. Both of these methods have their own characteristics, e.g. different spray particle velocities and flame temperatures. As a result, the coatings have different microstructures and properties (5).

\section{Experimental Coatings}

As aforementioned, electroplated chrome and four kinds of

*Acknowledgment, Thank you 1. Air Supply and Maintenance Center Command Eskisehir, Turkey for the A286 super alloy materials supplied and analysis facilities. plasma spray were involved in the study. Their characteristics are listed in Table 1. The A286 super alloy was used as substrate materials for all five coatings. The thicknesses of coating layers were controlled in the range of 100-150 $\mu \mathrm{m}$. The substrates were sand blasted prior to spraying using 36 grit alumina sand. Sulzer Metco 9MB plasma gun and GH / 732 nozzles were used. The spraying parameters were given in Table 2.

Table 1. The characteristics of the present coating materials.

\begin{tabular}{ccc}
\hline Designation & Composition $(w t . \%)$ & Powder size $(\mu \mathrm{m})$ \\
\hline Aluminum oxide & $\mathrm{Al}_{2} \mathrm{O}_{3} \% 3 \mathrm{TiO}_{2}$ & $-45+11$ \\
T400 & $\mathrm{Co}-28 \mathrm{Mo}-8 \mathrm{Cr}-2 \mathrm{Si}$ & $-45+15$ \\
Tungsten carbide & $\mathrm{WC} \% 12 \mathrm{Co}$ & $-45+15$ \\
Chrome carbide & $\% 75 \mathrm{Cr}_{3} \mathrm{C}_{2} \% 20 \mathrm{Ni} \% 5$ chromium & $-45+5$ \\
\hline
\end{tabular}

Table 2. Spray parameters.

\begin{tabular}{|c|c|c|c|c|}
\hline Coating Materials & $\begin{array}{l}\text { Aluminum } \\
\text { oxide }\end{array}$ & $\mathrm{T} 400$ & $\begin{array}{l}\text { Tungsten } \\
\text { carbide }\end{array}$ & $\begin{array}{l}\text { Chrome } \\
\text { carbide }\end{array}$ \\
\hline Plasma gases & $\mathrm{Ar}+\mathrm{H}_{2}$ & $\mathrm{Ar}+\mathrm{H}_{2}$ & $\mathrm{Ar}+\mathrm{H}_{2}$ & $\mathrm{Ar}+\mathrm{H}_{2}$ \\
\hline $\begin{array}{l}\text { Plasma gases flow } \\
\text { rates (scfh) }\end{array}$ & $100-15$ & $120-12,5$ & $110-12,5$ & $90-10$ \\
\hline $\begin{array}{l}\text { Plasma gases } \\
\text { pressure (psig) }\end{array}$ & $100-50$ & $90-50$ & $100-50$ & $100-50$ \\
\hline Current (amper) & 500 & 500 & 400 & 500 \\
\hline Spray distance (inch) & 4,5 & 4,5 & 4,5 & 4,5 \\
\hline Traverse speed & $\% 90-2$ & $\% 80$ & $\% 95$ & $\% 100$ \\
\hline $\begin{array}{l}\text { Powder feeder carrier } \\
\text { gas pressure (psig) }\end{array}$ & 50 & 50 & 50 & 50 \\
\hline $\begin{array}{l}\text { Powder feeder carrier } \\
\text { gas flow (scfh) }\end{array}$ & 13,5 & 13 & 13,5 & 15 \\
\hline Feed rate (g/min) & 50 & 45 & 30 & 50 \\
\hline Air jet & - & $\begin{array}{l}\text { Paralle, } \\
50 \text { psi }\end{array}$ & $\begin{array}{l}\text { Parallel, } \\
60 \text { psi }\end{array}$ & $\begin{array}{l}\text { Parallel, } 50 \\
\text { psi }\end{array}$ \\
\hline
\end{tabular}


Electroplated chrome coatings were produced in an industrial plant, using the industrial deposition parameters listed in Table 3. The de-hydrogenation thermal treatment $\left(200^{\circ} \mathrm{C}\right.$ for $\left.3 \mathrm{~h}\right)$ has also been performed on the coating.

\subsection{Characterization}

Roughness was measured with Diavite DH-5, also hardness off each coating were measured by the Vickers microhardness tester (Wilson/Tucon) and given in Table 4.

The thicknesses of the coatings were determined by micro hardness tester (Wilson/Tucon). Scanning electron microscopy technique (SEM) was used to observe two different parts of the test coupons which performed abrasive wear test (right) and which didn't (left).

\subsection{Abrasive Wear Tests}

For abrasive wear tests, samples were prepared from A286 with $4 \mathrm{~mm}$ thickness and $100 \mathrm{~mm}$ square, according to FED-STD141C. AMS 5525 (A286 plate form), electrolytic hard chrome and aluminum oxide, Co-28Mo- $8 \mathrm{Cr}-2 \mathrm{Si}$, tungsten carbide, chrome carbide plasma spray coated test panels were subjected to abrasive wear test. The wear tests were conducted with a Taber abraser, at room temperature, using a $1000 \mathrm{~g}$ load and CS-17 abrading wheel. The results were analyzed by wear index (mg/1000 cycles) and total wear (mg/10000 cycles) data. Cycles to $\mathrm{mg} / 1000$ weight loss is shown in Table 5 and Figure 1, cycles to total $\mathrm{mg}$ weight loss is given in Table $\mathbf{6}$ and Figure 2.

\section{Results and Comments}

In this study, heat treated electrolytic hard chrome and aluminum oxide, Co-28Mo- $8 \mathrm{Cr}-2 \mathrm{Si}$, tungsten carbide and chrome carbide plasma spray coated test coupons were characterized and abrasive wear behaviors were evaluated.

Table 3. Hard chrome deposition parameters.

\begin{tabular}{ll}
\hline Bath composition & $\begin{array}{l}\mathrm{CrO}_{3} 250 \mathrm{~g} / 1 ; \mathrm{H}_{2} \mathrm{SO}_{4} 2.5 \mathrm{~g} / \mathrm{l} ; \\
\text { no additives }\end{array}$ \\
\hline Bath temperature $\left({ }^{0} \mathbf{C}\right)$ & $52-57$ \\
Voltage $(\mathbf{V})$ & $2.5-3$ \\
Approximate current density $\left(\mathbf{A} / \mathbf{d m}^{2}\right)$ & $\approx 40$ \\
Bath stirring method & Pneumatic stirring \\
\hline
\end{tabular}

Table 4. Coating roughness and Vickers microhardness of coatings.

\begin{tabular}{ccccc}
\hline Coating & $\begin{array}{c}\text { Roughness; } \\
\mathbf{R}_{\mathbf{a}}(\boldsymbol{\mu m}) \\
(\mathbf{l t = 4 . 8} ; \\
\mathbf{l c = 0 . 8})\end{array}$ & $\begin{array}{c}\text { Standard } \\
\text { deviation }\end{array}$ & $\begin{array}{c}\text { Vickers } \\
\text { microhardness } \\
\text { (HV 0.1) }\end{array}$ & $\begin{array}{c}\text { Standard } \\
\text { deviation }\end{array}$ \\
\hline $\begin{array}{c}\text { Electrolytic } \\
\text { hard chrome } \\
\text { Aluminum } \\
\text { oxide }\end{array}$ & 144,2 & 16,87 & 662,27 & 4,56 \\
$\begin{array}{c}\text { Co-28Mo-8Cr-2Si } \\
\text { Tungsten carbide }\end{array}$ & 237,09 & 22,36 & 695,34 & 167,2 \\
Chrome carbide & 205,05 & 14,63 & 784,93 & 45,23 \\
\hline
\end{tabular}

Table 5. Abrasive wears weight loss (Cycles - weight loss, mg/1000).

\begin{tabular}{ccccccc}
\hline \multirow{2}{*}{ Cycles } & \multicolumn{6}{c}{$\mathbf{m g} / \mathbf{1 0 0 0}$} \\
\cline { 2 - 7 } & $\mathbf{A 2 8 6}$ & $\mathbf{K}^{\mathbf{1}}$ & $\mathbf{1 9}^{\mathbf{1}}$ & $\mathbf{3 6}^{\mathbf{1}}$ & $\mathbf{3 7}^{\mathbf{1}}$ & $\mathbf{3 8}^{\mathbf{1}}$ \\
\hline 0 & 0 & 0 & 0 & 0 & 0 & 0 \\
1000 & 0,02665 & 0,00958 & 0,0946 & 0,1405 & 0,22907 & 0,1655 \\
2000 & 0,0091 & 0,00647 & 0,05117 & 0,072 & 0,07357 & 0,0677 \\
3000 & 0,0054 & 0,00598 & 0,03313 & 0,0513 & 0,06627 & 0,0594 \\
4000 & 0,01705 & 0,0057 & 0,0317 & 0,0332 & 0,05997 & 0,0236 \\
5000 & 0,00995 & 0,0041 & 0,0281 & 0,0308 & 0,05827 & 0,0185 \\
6000 & 0,00475 & 0,00325 & 0,05883 & 0,05155 & 0,06223 & 0,0485 \\
7000 & 0,0167 & 0,0056 & 0,05597 & 0,0469 & 0,0543 & 0,02395 \\
8000 & 0,00685 & 0,00355 & 0,0508 & 0,0455 & 0,0442 & 0,0124 \\
9000 & 0,00715 & 0,00435 & 0,04853 & 0,0351 & 0,03363 & 0,0255 \\
10000 & 0,01385 & 0,00445 & 0,04703 & 0,04295 & 0,02333 & 0,0217 \\
\hline
\end{tabular}

Weight, mg,1000 Abraslve wears welght loss

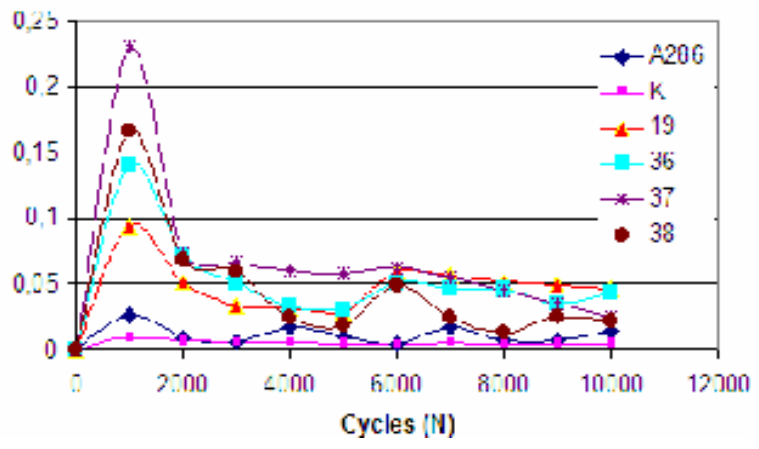

Figure 1. Abrasive wear weight loss vs. number of cycles.

Table 6. Abrasive wears weight loss (Cycles - Total weight loss, mg).

\begin{tabular}{ccccccc}
\hline \multirow{6}{*}{ Cycles } & \multicolumn{5}{c}{ Total mg } \\
\cline { 2 - 7 } & $\mathbf{A 2 8 6}$ & $\mathbf{K}^{\mathbf{1}}$ & $\mathbf{1 9}^{\mathbf{1}}$ & $\mathbf{3 6}^{\mathbf{1}}$ & $\mathbf{3 7}^{\mathbf{1}}$ & $\mathbf{3 8}^{\mathbf{1}}$ \\
\hline 0 & 0 & 0 & 0 & 0 & 0 & 0 \\
1000 & 0,02665 & 0,00958 & 0,0946 & 0,1405 & 0,22907 & 0,1655 \\
2000 & 0,03575 & 0,01605 & 0,14577 & 0,2125 & 0,30263 & 0,2332 \\
3000 & 0,04115 & 0,02203 & 0,1789 & 0,2638 & 0,3689 & 0,2926 \\
4000 & 0,0582 & 0,02773 & 0,2106 & 0,297 & 0,42887 & 0,3162 \\
5000 & 0,06815 & 0,03183 & 0,2387 & 0,3278 & 0,48713 & 0,3347 \\
6000 & 0,0729 & 0,03508 & 0,29753 & 0,37935 & 0,54937 & 0,3832 \\
7000 & 0,0896 & 0,04068 & 0,3535 & 0,42625 & 0,60367 & 0,40715 \\
8000 & 0,09645 & 0,04422 & 0,4043 & 0,47175 & 0,64787 & 0,41955 \\
9000 & 0,1036 & 0,04858 & 0,45283 & 0,50685 & 0,6815 & 0,44505 \\
10000 & 0,11745 & 0,05302 & 0,49987 & 0,5498 & 0,70483 & 0,46675 \\
\hline
\end{tabular}




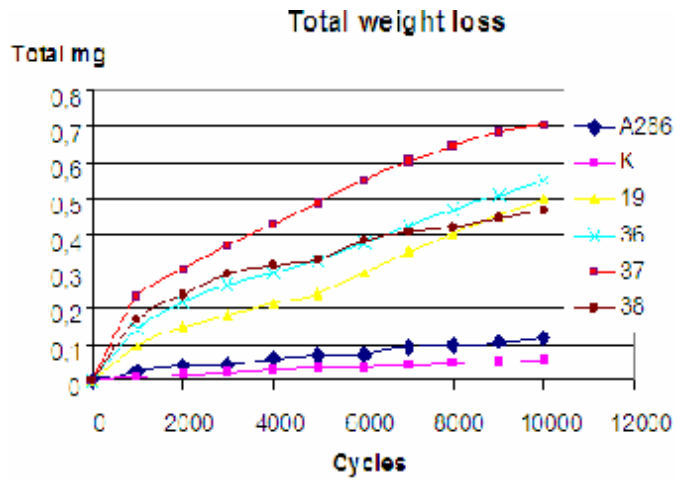

Figure 2. Total weight loss vs. number of cycles: $\mathrm{K}$ : Electrolytic hard chrome, 19 : Aluminum oxide, 36 : Co-28Mo-8Cr-2Si, 37 : Tungsten carbide, 38 : Chrome carbide.

Experimental data from abrasive wear tests were conclusive, indicating better results from the hard chrome coating. The abrasive wear resistance of plasma spray coatings and hard chromium plating was evaluated and the results in terms of wear weight loss are represented in Table 5, Table 6 and Figure 1, Figure 2. The hard chromium plating on A286 substrate material shows better wear resistance properties. Hard chromium coating reduces the abrasion rate of the substrate material.An initially higher wear weight loss for the plasma spray coatings occurred, decreasing continuously and then nearly stabilized. However, the stabilized abrasive wear rates were still higher than the hard chrome coatings.

The initial peak which is typical for plasma spray coatings (Figure 1) was due to the higher surface roughness. Table 4 figures out that the surface roughness values of all other coating materials are higher than those of electrolytic hard chrome. Plasma sprayed materials show rough surface properties, involving many pores, oxides and inclusions.

It is possible to compare the tested coating materials with electrolytic hard chrome coatings on the SEM micrographs which were given in Figure 3. It can clearly be observed that the electrolytic hard chrome coatings show dense and smooth surface properties. On the other hand, the plasma spray coatings have porous coating structure.
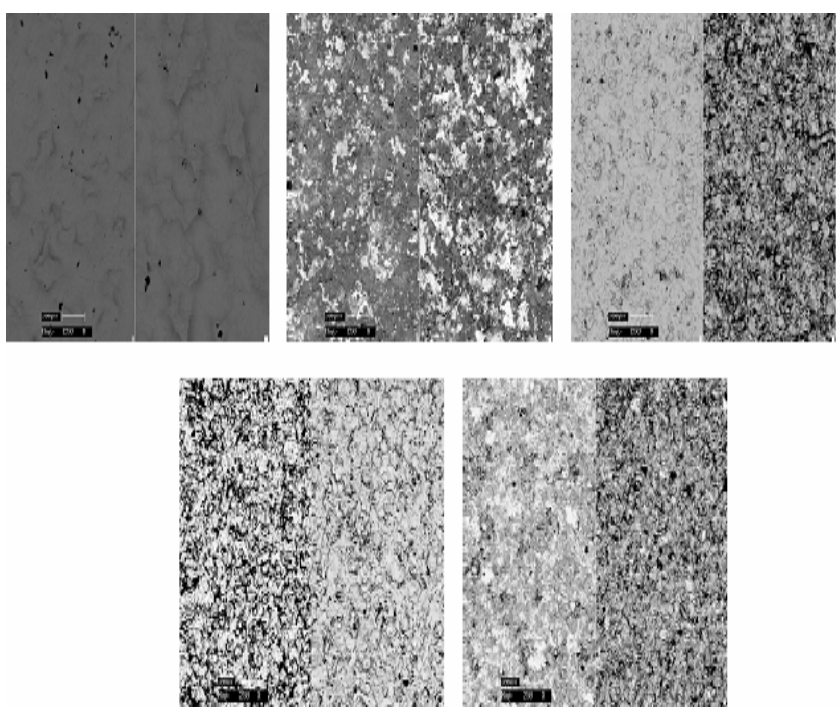

Figure 3. SEM micrographs of electrolytic hard chrome and plasma spray coatings (for each coating, left side picture non-weared, right side picture weared (From left to right electrolytic hard chrome, aluminum oxide, Co-28Mo-8Cr-2Si, tungsten carbide, chrome carbide) (250 $\mathbf{X})$ ).

In terms of hardness values, as it can be seen on Table 4, in comparison with the electrolytic hard chrome coated test coupons, similar or higher hardness values were reached by plasma spray coated test coupons.

As it can be seen in Table 5, Table 6 and Figure 1, Figure 2, aluminum oxide coatings show better abrasive wear resistance among all plasma spray coupons. This is due to high oxide content of the coating material. Coating of high oxide content is usually harder and is more wear resistant [6].

\section{REFERENCES}

[1] M.P. Nascimento, R.C. Souza, I.M. Miguel, W.L. Pigatin, H.J.C. Voorwald, Effects of tungsten carbide thermal spray coating by HP/HVOF and hard chromium electroplating on AISI 4340 high strength steel, Surface Coatings \& Technology, vol. 138, pp. 113-124, 2001.

[2] ASM handbook Volume 5: Surface Engineering, 1994.

[3] B.Navinsek, P.Panjan, I.Milosev, PVD coatings as an environmentally clean alternative to electroplating and electroless processes, Surface Coatings\&Technology, vol. 116-119 pp. 476-487, 1999

[4] L.Pawlowski, The Science and Engineering of Thermal Spray Coatings, John Wiley, New York, 1995.

[5] D.Toma, W. Brandl, G. Marginean, Wear and corrosion behaviour of thermally sprayed coatings, Surface Coatings \& Technology, vol. 138 pp. 149-158, 2001.

[6] H.J.C. Voorwald, R.C. Souza, W.L. Pigatin, M.O.H. Cioffi, Evaluation of WC-10Co-4Cr thermal spray coatings by HVOF on the fatigue and corrosion strength of AISI 4340 steel, Surface Coatings \& Technology, vol. 190, pp. 155-164, 2005. 